\title{
Comparison of maternal and neonatal outcome of IVF/ICSI conceived pregnancies with spontaneous conceived pregnancies
}

\author{
Preeti Patil $^{1}$, Gayathri Karthik ${ }^{2 *}$
}

\author{
${ }^{1}$ Department of Obstetrics and Gynecology, BLDE Medical College, Vijayapur, Karnataka, India \\ ${ }^{2}$ Department of Obstetrics and Gynaecology, Manipal Hospital, HAL Old Airport, Bangalore, Karnataka, India
}

Received: 13 September 2018

Accepted: 06 October 2018

\author{
*Correspondence: \\ Dr. Gayathri Karthik, \\ E-mail: gayathri.karthik@ manipalhospital.com
}

Copyright: (c) the author(s), publisher and licensee Medip Academy. This is an open-access article distributed under the terms of the Creative Commons Attribution Non-Commercial License, which permits unrestricted non-commercial use, distribution, and reproduction in any medium, provided the original work is properly cited.

\begin{abstract}
Background: Conception by IVF has been associated with an increased incidence of several obstetrical and perinatal complications. IVF is associated with increased complications like OHSS (Ovarian Hyper Stimulation Syndrome), multiple pregnancies and thromboembolism, ectopic pregnancy, congenital malformations, and small for gestational age, low birth weight etc. This study is done to evaluate and compare the obstetric and perinatal complications between assisted reproduction and spontaneous conception. The objective of the present study was to evaluate and compare the maternal and neonatal outcome of IVF/ICSI conceived pregnancies with spontaneous conceived pregnancies.

Methods: In this study 62 patients conceived with IVF/ICSI were taken as cases and 62 patients conceived spontaneously were taken as controls. The categorical data was analyzed using chi square test and results expressed by p value.

Results: The incidence of multiple pregnancies among IVF/ICSI group is $33.8 \%$ and $1.6 \%$ in controls. IVF/ICSI conception when compared to spontaneous conception is associated with increased incidence of miscarriage (18\% versus $1.6 \%$ ), first trimester bleeding (18\% versus $1.6 \%$ ), cervical insufficiency $(22.5 \%$ versus 0$)$, PROM (11.3\% versus $1.6 \%)$, preterm birth $(33.8 \%$ verss $14.5 \%)$, caesarean section rates $(71 \%$ vs $53 \%)$, low birth weight babies (44\% vs $10.7 \%$ ) and NICU admissions(33.3\% vs $10.7 \%$ ). The male: female ratio is higher in IVF/ICSI group.

Conclusions: IVF/ICSI conceptions are associated with a significantly higher incidence of multiple pregnancies, miscarriage, prematurity, increased caesarean section rates, and increased male: female ratio.
\end{abstract}

Keywords: IVF/ICSI, Obstetric and neonatal outcome, Spontaneous conception

\section{INTRODUCTION}

Simple assisted reproduction includes induction of ovulation, follicular scanning, and intrauterine insemination. If multiple cycles fail, if there is a tubal factor, or if there is a male factor etc, the patients are then recommended in vitro fertilisation and embryo transfer popularly referred to as IVF- ET. It is hence an important form of treatment for infertility when other methods of assisted reproductive techniques have failed. Conception by IVF has been associated with an increased incidence of several obstetrical and perinatal complications. Traditionally, IVF outcome has been expressed in terms of biochemical, clinical pregnancies or total live births. IVF is also associated with complications specific to itself like OHSS (ovarian hyperstimulation syndrome, multiple pregnancies and thromboembolic complications. There are reports to suggest an increased incidence of ectopic pregnancy, miscarriage, congenital malformations, and small for gestational age, low birth weight, preterm birth, stillbirth, perinatal death and increased rates of caesarean section. ${ }^{1-3}$ The precise 
reasons for this increase in adverse outcomes are not clear, but potential reasons include: maternal and paternal characteristics, underlying medical conditions associated with subfertility and infertility, sperm factors, the use of fertility medications, laboratory conditions during embryo culture, culture medium, cryopreservation and thawing, prenatal genetic diagnosis (if performed), differences in obstetrical management, increased proportion of multiple gestations and vanishing twins, or a combination of these factors. The effects of advanced maternal age also should not be underestimated, since many women who undergo IVF are older and older women are more likely to have relatively more pregnancy complications. $^{4}$

With a view to assess unbiasedly, the truth behind association between perinatal complications and assisted reproduction, the present study is being undertaken. In this study conducted at Manipal Hospital, Bangalore, the association between maternal and foetal outcomes in pregnancies following IVF/ICSI conceptions had been evaluated and compared with spontaneous conceptions.

\section{METHODS}

The present study is a prospective case control study conducted in the Department of Obstetrics and Gynaecology, Manipal Hospital, Bangalore between October 2013 and October 2014. In this study 62 patients conceived by IVF/ICSI as cases and 62 patients conceived spontaneously as controls were included. Permission for the study was obtained from the College authorities prior to commencement.

\section{Exclusion criteria}

- Women who have conceived with other forms of assisted reproductive techniques, with history of consanguineous marriage and patients with coexisting medical disorders (cardiac disease, pregestational diabetes, hypertension) were excluded from the study.

Patients were enrolled after taking the written informed consent. The demographic data such as age, occupation etc were obtained and detailed obstetrical, menstrual, medical, past history and also husband brief history entered in the profroma. These cases were followed up throughout the course of pregnancy, all complications like miscarriage, GDM, PIH, mode of delivery and neonatal complications were noted.

\section{Statistical analysis}

Observational and inferential statistical analysis has been carried out in the present study. The data obtained entered into Microsoft Excel worksheet and then analysed using chi square test and results expressed by $p$ value. Statistical significance was defined as a $\mathrm{p}$ value of less than or equal to 0.05 .

\section{RESULTS}

In the present study majority of women in both groups were in age group of 25-34 years. In IVF/ICSI pregnancies around $29 \%$ of women are above 35 years of age and $6.5 \%$ in control group Figure 1.

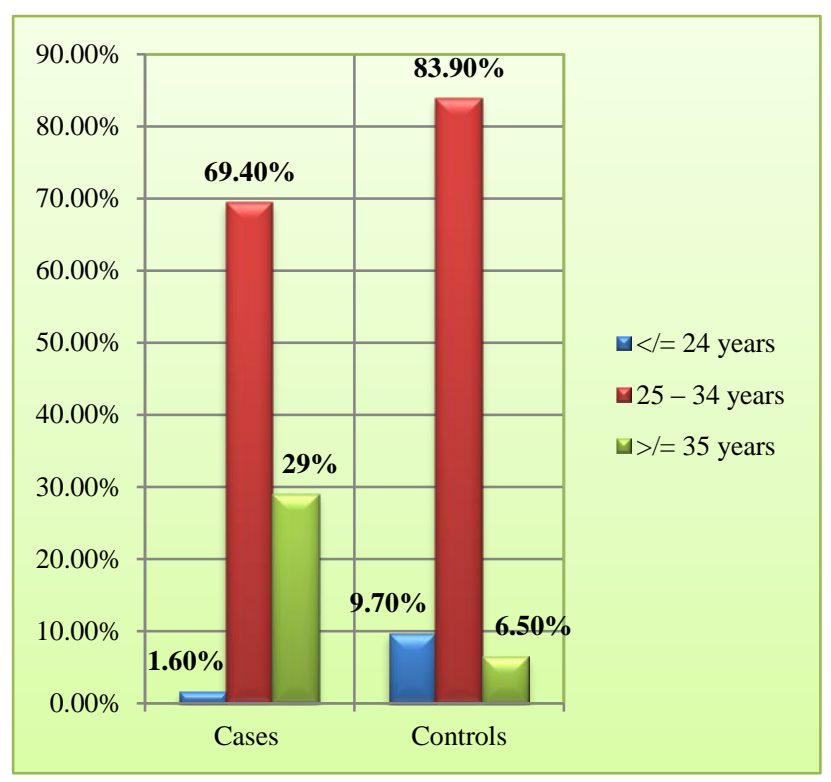

Figure 1: Comparison of age of patients.

In IVF/ICSI group $71 \%$ of women had primary infertility and $29 \%$ secondary infertility. $40.3 \%$ women in IVF/ICSI group had irregular cycles compared to $5 \%$ women in the control group Figure 2.

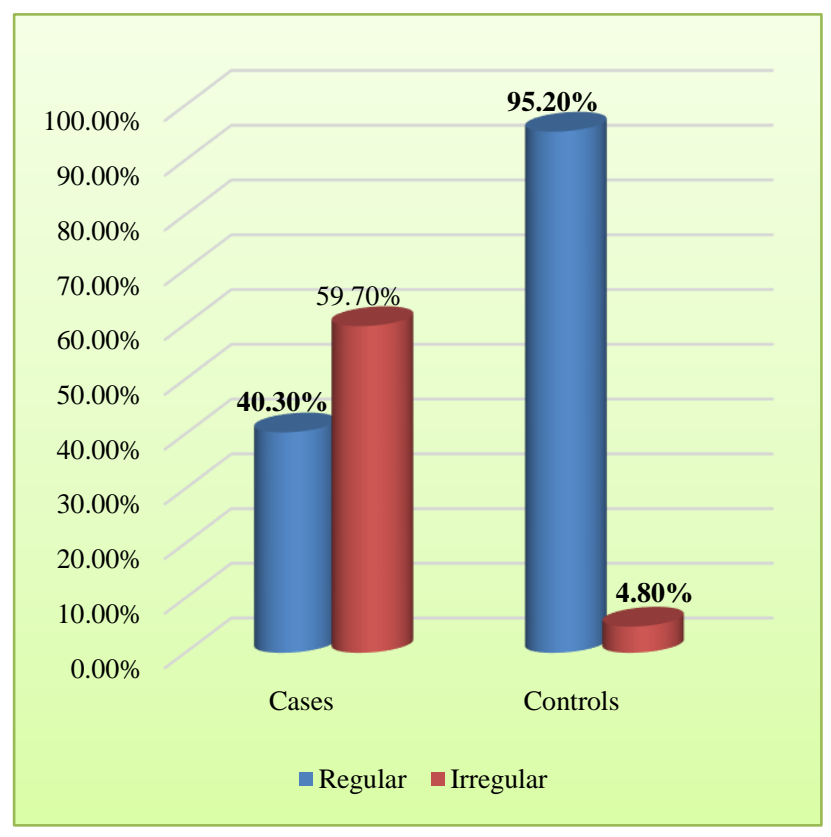

Figure 2 Comparison of menstrual cycles.

$29 \%$ of patients among IVF/ICSI group and $18 \%$ in controls were hypothyroid. Five patients among IVF 
group had of tuberculosis, 2 of them were treated cases of pulmonary tuberculosis and 3 had endometrial tuberculosis. 2 patients in each group had epilepsy. Polycystic ovarian syndrome (PCOS) is present in $46.7 \%$ of cases and $6 \%$ of controls ( $\mathrm{p}$ value-0.04). Amongst the cases $89 \%$ had increased BMI compared to $61 \%$ among controls. Among the indications for IVF/ICSI, $40.3 \%$ of patients had male factor, $32.2 \%$ had ovarian factor, $25.8 \%$ patients had tubal factor and $1.6 \%$ had uterine factor infertility. In the present study $81 \%$ of patients had 2 embryos transferred, $14.5 \%$ had $3,3.2 \%$ had single embryo transfer and only 1 patient had 4 embryos transfer. $40 \%$ of patients had IVF plus ICSI conception. $22.6 \%$ patients had frozen embryo transfer and $77.4 \%$ patients had fresh embryo transfer.

$63 \%$ amongst IVF/ICSI group were singleton pregnancies and $33.8 \%$ were multiple pregnancies ( $\mathrm{p}$ value-0.001) where as in controls $93.5 \%$ were singleton pregnancies and $1.6 \%$ had multiple pregnancies. In IVF/ICSI group, $95 \%$ of multiple pregnancies while $78 \%$ of singletons had obstetric complications. The miscarriage rate is $18 \%$ among IVF/ICSI group compared to $1.6 \%$ in control group ( $\mathrm{p}$ value $(0.02) .18 \%$ among cases and $1.6 \%$ among control group had first trimester bleeding $\mathrm{p}$ value $(0.01)$. Among IVF group $9.6 \%$ patients developed OHSS. 3 patients had mild, 2 had moderate and 1 had severe OHSS. Among IVF/ICSI group $22.5 \%$ had cervical insufficiency compared to none among the control group (pvalue-0.002). However, the rate of congenital malformation and gestational hypertension was similar in the two groups. GDM was present in $14.5 \%$ among cases compared to $8.1 \%$ among controls. The incidence of placenta previa was $6.4 \%$ in cases compared to $1.6 \%$ in controls. There were $11.3 \%$ patients of PROM among cases compared to $1.6 \%$ among controls ( $p$ value-0.02). $33.8 \%$ patients had preterm delivery among cases compared to $14.5 \%$ in controls. $71 \%$ of patients among IVF/ICSI had caesarean section compared to $53 \%$ among controls Table 1.

Table 1 Comparison of mode of delivery.

\begin{tabular}{|llll|}
\hline Mode of delivery & Cases $(\%)$ & Controls $(\%)$ & $\begin{array}{l}\text { P } \\
\text { value }\end{array}$ \\
\hline LSCS & $44(70.9)$ & $33(53.2)$ & 0.06 \\
\hline FTND & $01(01.6)$ & $21(33.8)$ & 0.001 \\
\hline Abortion & $11(17.7)$ & $01(1.6)$ & 0.003 \\
\hline Ectopic & $02(03.2)$ & $03(04.8)$ & 1.00 \\
\hline PTVD & $02(03.2)$ & $02(03.2)$ & 1.00 \\
\hline Hysterotomy & 00 & $01(01.6)$ & 1.00 \\
\hline $\begin{array}{l}\text { Induced MTP for } \\
\text { anamolus baby }\end{array}$ & $02(03.2)$ & $01(1.6)$ & 0.47 \\
\hline Total & $62(100)$ & $62(100)$ & \\
\hline
\end{tabular}

Among IVF/ICSI out of 66 babies born $21.2 \%$ were VLBW, $44 \%$ were LBW, $10.6 \%$ were SGA, $42.4 \%$ were of normal weights compared to $5.3 \%$ of VLBW, $10.7 \%$ were LBW, $7.2 \%$ were SGA and $76.8 \%$ were of normal weights among controls. There is no difference between the perinatal mortality between the two groups. The male: female ratio (1.53:1.07) was more among IVF/ICSI group compared to controls. There were 2 neonatal deaths in each group. The overall complication was higher among IVF/ICSI group compared to controls. Higher maternal age, nulliparity is seen in IVF/ICSI group. There is higher incidence of miscarriage, first trimester bleeding, cervical insufficiency, PROM and preterm deliveries and VLBW and LBW in IVF/ICSI group as compared to controls. Male: female ratio is higher among IVF/ICSI group. Table 2.

Table 2 Comparison of neonatal complications.

\begin{tabular}{|c|c|c|c|}
\hline $\begin{array}{l}\text { Neonatal } \\
\text { complications }\end{array}$ & $\begin{array}{l}\text { Cases } \\
(\%)\end{array}$ & $\begin{array}{l}\text { Controls } \\
(\%)\end{array}$ & $\begin{array}{l}\mathbf{P} \\
\text { value }\end{array}$ \\
\hline No. of complications & 69 & 31 & 0.04 \\
\hline Preterm & $21(30.4)$ & $09(29.1)$ & 0.43 \\
\hline Jaundice & $08(11.6)$ & $04(13.3)$ & 0.36 \\
\hline Low birth weight & $17(24.6)$ & $10(33.3)$ & 0.12 \\
\hline $\begin{array}{l}\text { Very low birth } \\
\text { weight }\end{array}$ & $8(11.6)$ & $03(10)$ & 0.20 \\
\hline $\begin{array}{l}\text { Respiratory distress } \\
\text { syndrome }\end{array}$ & $06(08.7)$ & $02(6.6)$ & 0.27 \\
\hline GERD & $02(02.8)$ & 00 & 0.47 \\
\hline Sepsis & $01(01.4)$ & 00 & 1.00 \\
\hline Anemia & $01(01.4)$ & 00 & 1.00 \\
\hline TTN & $02(02.8)$ & $02(6.6)$ & 1.00 \\
\hline ROP & $03(04.3)$ & 00 & 0.24 \\
\hline SGA & 00 & $01(3.3)$ & 1.00 \\
\hline
\end{tabular}

\section{DISCUSSION}

The incidence of infertility is around $10-15 \%$. These couples require some form of assistance in conception, which may be in the form as minimal as ovulation induction or as intensive as IVF/ICSI. The number of pregnancies as a result of IVF/ICSI procedures exceeds 3 million in the world. Although most pregnancies after IVF result in normal healthy outcomes, an increased risk for a number of obstetric and neonatal complications, compared to naturally conceived pregnancies, has been variably reported. ${ }^{5}$

Increasing maternal age is a risk factor for obstetric and perinatal complications. The average age at which women attempt to conceive continues to rise, due to more women putting profession ahead of conceiving. Also, women who have had difficulty in conceiving are also older by the time they come for treatment with IVF. Older women are already predisposed to age related complications like GDM, hypertension etc.

Irregular cycles are more commonly seen in PCOS women due to oligo-ovulation and anovulation as a result of which the need for ART arises. PCOS is the most common endocrine syndrome affecting women of reproductive age with a prevalence of between $4-18 \%$ generally and up to $50 \%$ in South Asian women. ${ }^{6}$ PCOS 
is more prevalent in obese women than those who are lean, affecting around $28 \%$ versus $5 \%$ respectively (p0.04 ). It substantially contributes to infertility; in fact, it accounts for $90-95 \%$ of women attending infertility clinics with anovulatory cycles (Balen, RCOG. PCOS women are at increased risk of miscarriage, GDM, PIH, preterm-birth and perinatal mortality. ${ }^{7,8}$

Initially, infertility was considered primarily a female problem and male infertility is often overlooked. The male infertility is on rising trend as seen in the study done by Hull MG et al, the percentage of male infertility was $32 \%$, but in the study done by Manish B et al shows an incidence of about $40 \% .^{9,10}$ Also present study showed $40 \%$ of male infertility, as a result male infertility cannot be neglected. In the present study the ovarian cause of infertility is also higher, which may due to decreased ovarian reserve in older women and increased incidence of PCOS due to unhealthy lifestyle. The incidence of tubal factor infertility is also increasing due to the increased prevalence of tuberculosis, PID and endometriosis. Increased incidence of PID is attributable to promiscuity. Genital tuberculosis accounts for $1-19 \%$ of infertility cases in India. ${ }^{11}$ Incidence is still higher in women seeking assisted reproduction being $24.5 \%$ overall but as high as $48.5 \%$ with tubal factor infertility is reported by Singh et al. ${ }^{12}$

With the development of ART, the advent of ovarian stimulation to produce multiple oocytes and the transfer of multiple embryos in order to improve pregnancy and live birth rates, there has been an increase in the rate of multiple pregnancies. The incidence of multiple pregnancies is comparable to the study done by Gunby $\mathbf{J}$ et al and others. ${ }^{13}$ Following IVF/ICSI conception the miscarriage rate is higher than the spontaneous conception, this may be attributable to increased maternal age (Farr SL et al14), PCOS, hypothyroidism, overweight or obesity (Maheshwari A et al) etc. Both women's age and increased BMI have detrimental effect, but the same is observed in fertile population. ${ }^{14,15}$ The influence of the cause of infertility on miscarriages is also unclear.

Compared to spontaneously conceived pregnancies, IVF group had more miscarriage rate as similar to the study done by Philippe $\mathrm{T}$ et al (17.2\%). IVF/ICSI conceived pregnancies are at high risk for first trimester bleeding. ${ }^{16}$ The reason of the above is unknown, however the influence of ovarian stimulation, luteal insufficiency and spontaneous embryo reduction are suggested. The technique of fertilization (IVF or ICSI) does not seem to be relevant (Ochsenkuhn $\mathrm{R}$ et al). ${ }^{17}$ The study by Petra De et al also showed higher incidence of about $17.6 \%$, other studies showed much higher incidence than the present study $(36.2 \%$ in Hofmann et al, $31.2 \%$ in Pezeshki et al. ${ }^{18-20}$ It has been shown that first-trimester bleeding is correlated with an increased risk for miscarriage both in spontaneous (50\% in Everett et al) and in IVF pregnancies. But in the present study only one patient had miscarriage after first trimester bleeding among the IVF/ICSI group. ${ }^{21}$

Ectopic pregnancy rates have raised over the past decade although the reasons are unclear, improved and early diagnosis certainly has an effect It is suggested that the history of PID (pelvic inflammatory disease), tubal factor of infertility and embryo transfer technique can lead to increased incidence of ectopic pregnancy. OHSS (Ovarian hyperstimulation syndrome) is iatrogenic complication, unique to IVF, following ovarian stimulation. The incidence of mild forms of OHSS are common, affecting up to $20-33 \%$ of in vitro fertilisation (IVF) cycles, 3-6\% of IVF cycles are complicated by moderate and $0.1-2 \%$ by severe OHSS. ${ }^{22}$ OHSS is more commonly found in women with PCOS and with fresh embryo transfer group due to hormonal stimulation. Half of women who developed OHSS had PCOS among IVF/ICSI group.

Among IVF/ICSI group $22.5 \%$ had cervical insufficiency compared to none among the control group ( $\mathrm{p}$ value0.002 ) which is similar to the study done by Kazem et al $(22.5 \%) .^{23}$ Increased operative interventions in the IVF/ICSI group may have led to the increased incidence of cervical insufficiency. Current information shows that IVF/ICSI pregnancies are associated with 1.5-fold increased risk of congenital abnormality (Ates and Buckett). ${ }^{24}$ However it is unclear, whether this small increased risk is related to infertility itself or its treatment. In the present study, the rate of congenital malformation was similar in the two groups. The study done by Hansen et al and others also showed no significant statistical difference between case and controls. $^{25}$

There is reportedly an increased risk obstetric complication like GDM, pre-eclampsia, abruption, placenta praevia in IVF/ICSI group. The increased complications may be attributable to multiple pregnancies, older age group etc. The placenta praevia aetiology remains unclear, but several studies have reported higher frequencies of placenta previa in pregnancies of women with advanced maternal age, multiparity and previous caesarean delivery or abortion. In a meta-analysis of complications related to assisted reproduction, the investigators reported three-fold higher risk of placenta previa associated with the treatment (Jackson et al). ${ }^{26}$ Increased caesarean section rates may be attributed to the increased associated complications, precious nature of pregnancy and anxiety on the part of the couples among cases.

Neonatal morbidity is higher among IVF/ICSI twin compared to singleton. However, IVF/ICSI singleton also shows higher incidence of preterm birth. The main reason for the morbidity is prematurity which leads to low birth weight babies, and increased rates of NICU admission. The increased incidence of preterm in IVF/ICSI pregnancies may be caused by the IVF technique per se, 
the ovarian stimulation, or the infertility. The prevalence of preterm birth in the IVF group is almost 3 times higher than the control group. The difference between the two groups is statistically significant. The study done by Hung Chieh $\mathrm{C}$ et al also showed higher incidence of preterm birth in IVF pregnancies. It is commonest cause of perinatal morbidity. ${ }^{27}$ The incidence among control group also showed higher incidence of preterm birth this is due to increased maternal complications like PIH, GDM which had led to preterm delivery at our hospital.

Multiple pregnancies contribute to almost one-third of all pregnancies conceived by IVF/ICSI. It constitutes almost $80 \%$ of preterm births among IVF group. Compared to singletons pregnancies multiple pregnancies have more number of low birth weight babies and thus more number of NICU admissions. The higher incidence of male babies after IVF may be attributable to the fact that male embryos divide faster than female embryos and selection of embryo is based on degree of cleavage.

\section{CONCLUSION}

Increased maternal age, nulliparity and obesity are more commonly seen in women undergoing IVF/ICSI pregnancies, all of which predispose to complications. Miscarriage rate is higher among IVF/ICSI conception. PCOS women are at increased risk of developing OHSS. IVF conceptions are associated with a significantly higher (one-third) incidence of multiple pregnancies. Prematurity is 3 times more in IVF/ICSI group, hence low birth weight babies and NICU admission rates is more common among IVF group. There is no difference in the incidence of congenital malformation between both groups. Perinatal mortality is not increased with IVF/ICSI conception. The male: female ratio is higher in IVF/ICSI conception.

\section{ACKNOWLEDGMENTS}

Authors would like to thank all the participants for their co-operation and support during the study.

Funding: No funding sources

Conflict of interest: None declared

Ethical approval: The study was approved by the Institutional Ethics Committee

\section{REFERENCES}

1. Forman RG, Frydman R, Egan D, Ross C, Barlow DH. Severe ovarian hyperstimulation syndrome using agonists of gonadotropin-releasing hormone for in vitro fertilization: a European series and a proposal for prevention. Fertil Steril. 1990;53(3):502-9.

2. Lesny P, Killick SR, Robinson J, Maguiness SD. Transcervical embryo transfer as a risk factor for ectopic pregnancy. Fertil Steril. 1999;72(2):305-9.
3. Klemetti R, Gissler M, Sevón T, Koivurova S, Ritvanen A, Hemminki E. Children born after assisted fertilization have an increased rate of congenital anomalies. Fertil Steril 2005;84(5):13007.

4. Sperooff L, Fritz MA. Assisted reproductive technologies. Clinical Gynecologic and endocrinology and infertility $2014 ; 8^{\text {th }}$ ed:1331-81.

5. Raatikainen K, Kuivasaari-Pirinen P, Hippeläinen M, Heinonen S. Comparison of the pregnancy outcomes of subfertile women after infertility treatment and in naturally conceived pregnancies. Hum Reprod 2012; 27(4):1162-9.

6. McFarland C. Treating polycystic ovarian syndrome and infertility. MCN Am J Matern Child Nurs. 2012;37(2):116-21.

7. Balen et al. Managing anovulatory infertility and polycystic ovary syndrome. BMJ 2007 Sep 29; 335(7621):663-6.

8. Sunita R Tandulwadkar. Obstetric complications in women with IVF conceived pregnancies and polycystic ovarian syndrome. J Hum Reprod Sci. 2014;7(1):13-8.

9. Manish B, Patel PM, Basic management of infertility. Principles and Practice of Obstetrics and Gynaecology for postgraduates. FOGSI pub 2008; $3^{\text {rd }}$ ed, 2:723-30.

10. Hull MG, Glazener CM, Kelly NJ, Conway DI, Foster PA, Hinton RA. Population study of causes, treatment, and outcome of infertility. Br Med J (Clin Res Ed). 1985;291(6510):1693-7.

11. Gupta N, Sharma JB, Mittal S, Singh N, Misra R, Kukreja M. Genital tuberculosis in Indian infertility patients. Int J Gynnecol Obstet 2007;97(2):135-8.

12. Singh N, Sumana G, Mittal S. Genital tuberculosis: a leading cause of infertility in women seeking assisted conception in North India. Arch Gynecol Obstet 2008;278(4):325-7.

13. Gunby J, Bissonnette F, Librach C, Cowan L. Assisted reproductive technologies (ART) in Canada: 2007 results from the Canadian ART Register. Fertil Steril 2011;95(2):542-7.e1-10.

14. Farr SL, Schieve LA, Jamieson DJ. Pregnancy loss among pregnancies conceived through assisted reproductive technology, United States, 1999-2002. Am J Epidemiol 2007;165(2):1380-8.

15. Maheshwari A, Stofberg L, Bhattacharya S. Effect of overweight and obesity on assisted reproductive technology a systemic review. Hum Reprod Update 2007;13(5):433-44.

16. Tummers P, Sutter PD, Dhont M. Risk of spontaneous abortion in singleton and twins pregnancies after IVF/ICSI. Hum Reprod. 2003;18(8):1720-3.

17. Ochsenkühn R, Strowitzki T, Gurtner M, Strauss A, Schulze A, Hepp H, et al. Pregnancy complications, obstetric risks, and neonatal outcome in singleton and twin pregnancies after GIFT and IVF. Arch Gynecol Obstet. 2003. 268(4):256-61. 
18. De Sutter P, Bontinck J, Schutysers V, Van der Elst J, Gerris J, Dhont M. First-trimester bleeding and pregnancy outcome in singletons after assisted reproduction. Human Reprod. 2006;21(7):1907-11.

19. Hofmann GE, Gundrum CL, Drake LM, Bertsche AB. Frequency and effect of vaginal bleeding on pregnancy outcome during the first 3 weeks after positive B-hCG test results following IVF-ET. Fertil Steril 2002;74(3):609-10.

20. Pezeshki K, Feldman J, Stein DE, Lobel SM, Grazi RV. Bleeding and spontaneous abortion after therapy for infertility. Fertil Steril 2000;74(3):504.

21. Everett C. Incidence and outcome of bleeding before 20th week of pregnancy: prospective study from general practice. BMJ 1997;315(7099):32-4.

22. Pandey S, Shetty A, Hamilton M, Bhattacharya S, Maheshwari A. Obstetric and perinatal outcomes in singleton pregnancies resulting from IVF/ICSI: a systematic review and meta analysis. Human reproduction update 2012;18(5):485-503.

23. Nouri K, Ott J, Stoegbauer L, Pietrowski D, Frantal $\mathrm{S}$, Walch K. Obstetric and perinatal outcomes in IVF versus ICSI-conceived pregnancies at a tertiary care center-a pilot study. Reproduct Biol Endocrinol. 2013;11(1):84.
24. Buckett A. Obsteric and neonatal outcome following assisted reproduction. Current Prog Obstet. Gynaecol. 2012;1:98-117.

25. Hansen M, Kurinczuk JJ, Bower C, Webb S.The risk of major birth defects after intracytoplasmic sperm injection and invitro fertilization. $\mathrm{N}$ Engl J Med 2002;346(10):725-30.

26. Jackson RA, Gibson KA, Wu YW, Croughan MS. Perinatal outcomes in singletons following in vitro fertilization: a meta-analysis. Obstet Gynecol 2004;103(3):551-63.

27. Chou HC, Tsao PN, Yang YS, Tang JR, Tsou KI. Neonatal outcome of infants born after in vitro fertilization at National Taiwan University hospital. J Formos Med Assoc. 2002;101:203-5.

Cite this article as: Patil P, Karthik G. Comparison of maternal and neonatal outcome of IVF/ICSI conceived pregnancies with spontaneous conceived pregnancies. Int J Reprod Contracept Obstet Gynecol 2018;7:4715-20. 